\title{
Anticoagulation Therapy Considerations in Factor VII Deficiency
}

\author{
Eric Paulus ${ }^{1} \cdot$ Kathy Komperda $^{2} \cdot$ Gabriel Park $^{2} \cdot$ Julie Fusco $^{2}$
}

Published online: 8 August 2016

(c) The Author(s) 2016. This article is published with open access at Springerlink.com

\begin{abstract}
Factor VII (FVII) deficiency is the most prevalent rare bleeding disorder in the USA and affects approximately 1 out of every 500,000 people. Warfarin inhibits the synthesis of FVII, in addition to other clotting factors. Warfarin is contraindicated in patients with bleeding tendencies or blood dyscrasias; therefore, the literature regarding the use of warfarin in FVII deficiency is very limited. We report a successful re-challenge of warfarin therapy in a patient with FVII deficiency. A 70-yearold woman with FVII deficiency experienced a significant decrease in FVII activity and subsequent vaginal bleeding roughly 5 weeks after starting warfarin for atrial fibrillation. The patient was switched to aspirin therapy. Nearly 4 years later, warfarin therapy was re-attempted by a different haematologist. After 9 months, FVII activity remained in an acceptable range and no bleeding events had occurred. In addition, once the maintenance dose was established, the international normalized ratio remained within the goal range (1.5-2.0) for the majority of assessments. Regarding future considerations, we hypothesize that anticoagulants that do not directly affect FVII, such as the direct oral anticoagulants, would carry less risk of bleeding complications and therefore may be safer alternatives to warfarin to reduce the risk of thromboembolic stroke in patients with atrial fibrillation and FVII deficiency.
\end{abstract}

Eric Paulus

eric.paulus@advocatehealth.com; ejpaulus@gmail.com

Advocate Medical Group, Chicago, IL, USA

2 Midwestern University Chicago College of Pharmacy, 555 31st Street, Downers Grove, IL 60515, USA

\section{Key Points}

We observed a successful re-challenge of warfarin therapy in a patient with atrial fibrillation and factor VII (FVII) deficiency.

Challenges with using warfarin therapy in FVII deficiency include lack of a defined optimal target international normalized ratio range and a poor correlation between the degree of FVII deficiency and bleeding risk.

We hypothesize anticoagulants which do not directly affect FVII, such as the direct oral anticoagulants, may carry less risk of bleeding complications than warfarin to reduce the risk of thromboembolic stroke in patients with atrial fibrillation and FVII deficiency.

\section{Introduction}

The coagulation pathway is a complex system and is an essential part of haemostasis [1]. It involves a number of enzymatic steps, which activate circulating clotting factors, ultimately leading to clot formation (see Fig. 1). Coagulation is typically initiated through the extrinsic pathway when tissue injury exposes tissue factor. Circulating factor VII (FVII) binds to tissue factor, and the resulting complex becomes activated FVII (FVIIa). FVIIa then directly catalyses the conversion of factor $\mathrm{X}$ to factor $\mathrm{Xa}$ $(\mathrm{FXa})$ in the common pathway and indirectly via the 
Fig. 1 Effects of multiple anticoagulant medications on the coagulation cascade

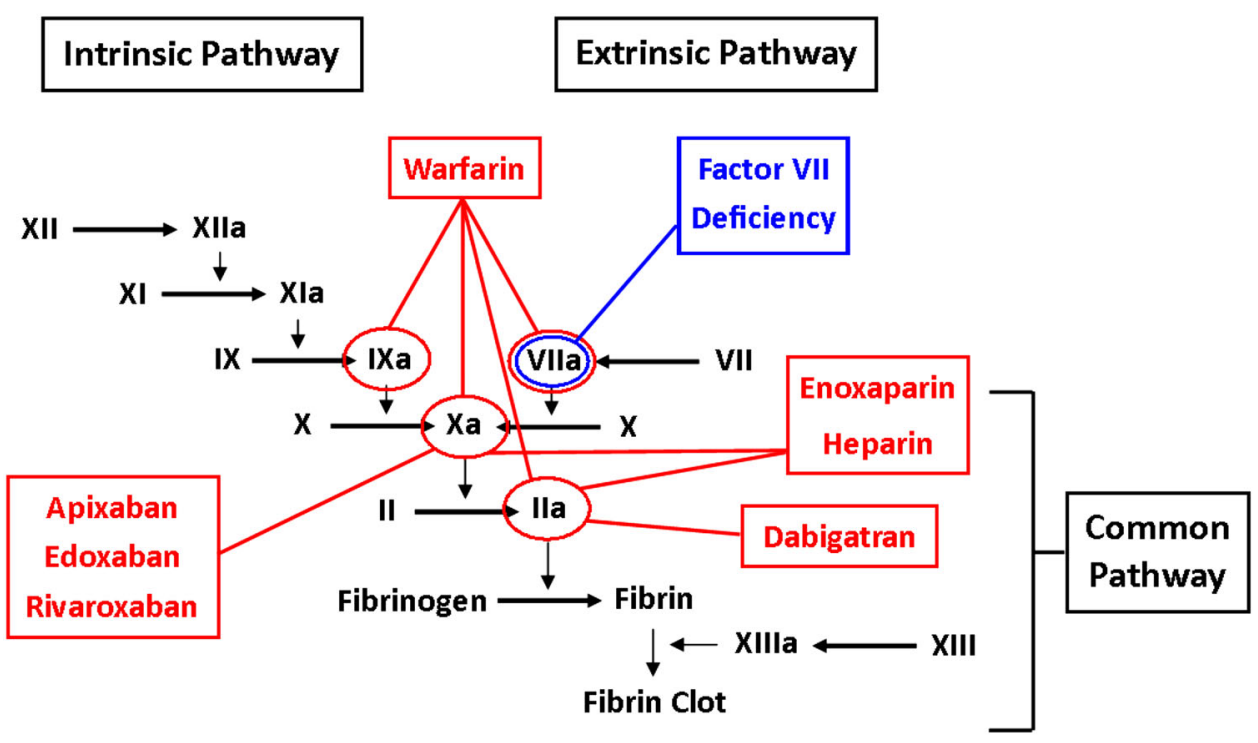

activation of factor IX to factor IXa. While the extrinsic pathway initiates haemostasis, sustained haemostasis is dependent upon the continued and amplified procoagulant action of the intrinsic pathway, which involves factors VIII, IX, XI and XII [2, 3].

Given the complex nature of the coagulation pathway, one can appreciate the complexity of coagulation disorders. Classical bleeding disorders include haemophilia A and B; however, a number of rare bleeding disorders (RBDs) exist [4]. RBDs represent only about 3-5\% of inherited coagulation deficiencies and include deficiencies in fibrinogen; factors II, V, V + VIII, VII, X and XIII; and a combination of the vitamin K-dependent factors [5]. The most prevalent RBD is FVII deficiency, which affects approximately 1 out of every 500,000 people in the USA, and the prevalence varies in other countries [6]. FVII deficiency is an autosomal-recessive bleeding disorder. The hallmark of FVII deficiency is a prolonged prothrombin time (PT) and an elevated international normalized ratio (INR) in the setting of normal liver function and a normal activated partial thromboplastin time (aPTT) [7].

Managing an FVII-deficient patient presents several challenges. First, FVII can potentially exist in several different forms, and measuring FVII is further complicated by the many different assays available [8-10]. For the purposes of this article, FVII is reported as FVII coagulation activity (FVIIc), expressed as a percentage, with normal activity being $50-150 \%$. Our institution utilized a one-step clotting assay, with the result being normalized to determine the percentage activity. Second, determining disease severity on the basis of FVIIc is also difficult, as there is a poor correlation between FVIIc and bleeding risk, as some patients with moderate to severe deficiency may remain asymptomatic, while others with mild deficiency may experience major bleeding events [11]. Disease severity may be better classified on the basis of the location and frequency of bleeding events [7, 12]. A recent retrospective analysis of 83 patients with FVII deficiency undergoing surgical procedures recommended using FVII levels, the bleeding history and the type of surgery to help estimate bleeding risk and guide perioperative management [13]. Lastly, gene analysis is also difficult to interpret, as there are over 130 known mutations, with many still lacking defined phenotypic characteristics [11]. Progress is being made as national and international registries for RBDs continue to provide information about FVII deficiency.

Although RBDs inherently limit coagulation and may predispose patients to bleeding, patients with FVII deficiency may still require anticoagulation therapy if indicated. A case series analysed thromboembolic events in patients with FVII deficiency and concluded that those with mild FVII deficiency should not be precluded from receiving antithrombotic prophylaxis [14]. Historically, warfarin has been the initial treatment of choice in this limited patient population. Warfarin works by blocking the regeneration of vitamin $\mathrm{K}$ epoxide, thus inhibiting the synthesis of vitamin K-dependent clotting factors II, VII, IX and $\mathrm{X}$, as well as the anticoagulant proteins $\mathrm{C}$ and $\mathrm{S}$. There is evidence to suggest that not all of the vitamin Kdependent clotting factors are affected equally by warfarin, with FVII and factor IX being suppressed to a greater degree than factors II and X [15]. The PT is used to monitor warfarin therapy and is converted to an INR for standardization. The PT reflects the functionality of several different clotting factors, including the vitamin K-dependent clotting factors; however, a prolonged PT is not seen until one of the clotting factors has decreased to $<30-40 \%$ [16]. For conventional patients who are stable on warfarin 
therapy, the INR should accurately represent variations in coagulation factor activities [17]. However, in the presence of FVII deficiency, the INR may not as accurately represent the degree of coagulation ability, given the inherent decrease of FVII in this RBD. Monitoring treatment through FVII activity may also prove to be difficult, as the minimal levels needed to sustain haemostasis are not well established [7]. There is limited published literature to guide anticoagulation therapy and monitoring in this population due to the rare nature of the disorder.

Three case reports involving the use of vitamin $\mathrm{K}$ antagonists for atrial fibrillation in patients with FVII deficiency have been published:

- The first case was a 50-year-old male with a prolonged PT and an INR of 1.5 at baseline [18]. He developed an INR of 19.6 after four doses of warfarin $5 \mathrm{mg}$, which prompted discontinuation. Two weeks later, laboratory analysis revealed an FVIIc of $43 \%$, which led to the diagnosis of mild FVII deficiency. Warfarin was restarted at a higher INR goal of 2.5-3.5 to ensure adequate anticoagulation in the presence of an elevated baseline INR. After 18 months, no bleeding or thrombotic events were reported, despite the INR being in the therapeutic range only $32 \%$ of the time.

- The second case was a 77-year-old female [19]. She developed an INR of 6.0 after two doses of warfarin $10 \mathrm{mg}$, which prompted discontinuation. Thirteen days later, her INR was 2.2. Warfarin was re-started, and over the next few months, the patient's INR ranged from 2.2 to 3.8 with doses ranging from 0.5 to $4 \mathrm{mg}$ weekly. When warfarin was withheld for 2 weeks and the patient's INR remained elevated at 3.5, she was evaluated for an RBD. Laboratory analysis revealed an FVIIc of $14 \%$. It was concluded that the risks with warfarin outweighed the benefits, and the patient was switched to aspirin.

- The third case was an 80-year-old male with a baseline INR of 2.1 and an FVIIc of $10 \%$ [20]. His INR goal was set at 2.5-3.0. While his INR was within the goal range, his FVIIc decreased to $5 \%$ (equal to a $50 \%$ decrease from baseline), whereas none of the other vitamin K-dependent clotting factors decreased more than $30 \%$ in comparison with baseline. Given the risk of bleeding and perceived inadequate anticoagulant effect, warfarin was discontinued, and enoxaparin (a low molecular weight heparin [LMWH]) was initiated, which was monitored using anti-FXa activity. The patient received enoxaparin for 3 months without any bleeding symptoms before undergoing successful cardioversion.

In summary, although none of these patients developed major bleeding symptoms with vitamin $\mathrm{K}$ antagonist therapy, all encountered challenges with monitoring and accurate assessment of the anticoagulant effect. Such challenges lead to uncertainties in how to implement anticoagulation therapy in patients with this RBD, as further highlighted by the case summary below.

\section{Case Report}

A 70-year-old African American female with atrial fibrillation was referred to haematology in 2010 for evaluation of a mildly prolonged PT with persistent vaginal bleeding. Laboratory test results revealed a decreased FVIIc of $33 \%$ (reference 50-150 \%), and FVII deficiency was diagnosed. The patient underwent endometrial cryotherapy, and after 4 months without recurrent vaginal bleeding, haematology cleared the patient to start warfarin therapy, with an INR goal of 2.0-3.0. Haematology recommended discontinuing warfarin if FVIIc decreased to $10 \%$ or less. The patient's baseline INR was 1.4 , and warfarin was conservatively started at $2 \mathrm{mg}$ daily. The INR remained at 1.4 for the first 4 weeks of therapy, despite gradual dose increases. Nearly 6 weeks into therapy, the patient made a dosing error, which caused her INR to increase to 2.5. She developed recurrent vaginal bleeding, and her FVIIc was found to be $10 \%$, which prompted warfarin discontinuation. She was switched to aspirin $81 \mathrm{mg}$ daily.

In 2014, she was evaluated by a different haematologist. Her FVIIc was $44 \%$, with a baseline INR of 1.2. It was decided to re-attempt warfarin therapy with a lower INR goal of 1.5-2.0, as the risks of stroke without anticoagulation seemed to outweigh the risks of bleeding with anticoagulation. The patient's risk factors for stroke had not changed since her initial warfarin course. Haematology recommended discontinuing warfarin if FVIIc decreased to $<15 \%$ or if the patient developed any clinical signs of bleeding. A total weekly dose of $40 \mathrm{mg}$ was found to maintain her INR in the range of 1.6-1.8. The FVIIc varied between 24 and $28 \%$ during the first 6 months of warfarin therapy. During the seventh month, the patient's FVIIc decreased to $15 \%$, with a corresponding increase in her INR to 2.4 , despite no change in her weekly dose. The patient denied any signs of bleeding during this second treatment course. Her weekly doses, INR results and FVIIc results are shown in Table 1.

\section{Discussion}

Our case report describes a successful re-challenge of warfarin therapy in a patient with FVII deficiency. However, we must recognize the varying degrees of FVIIc and the corresponding bleeding risk among patients with FVII 
Table 1 Case patient dose, international normalized ratio (INR) and factor VII coagulation activity (FVIIc) results

\begin{tabular}{lllll}
\hline Date & Dose (mg/week) & INR & FVIIc (\%) & Comments \\
\hline September 27, 2010 & None & 1.3 & 33 & First haematology consultation \\
April 11, 2011 & None & 1.4 & - & Warfarin 2 mg daily started-1st attempt \\
May 5, 2011 & 24 & 1.4 & 37 & \\
May 23, 2011 & 56 & 2.5 & 10 & Dose error, recurrent vaginal bleeding, warfarin \\
& & & & discontinued \\
March 26, 2014 & None & 1.2 & 44 & Wecond haematology consultation \\
June 19, 2014 & None & 1.2 & - & \\
August 11, 2014 & 32 & 1.4 & 24 & \\
October 7, 2014 & 40 & 1.7 & 28 & mo daily started-2nd attempt \\
December 11, 2014 & 40 & 1.7 & 32 & No signs/symptoms of bleeding, warfarin dose \\
January 27, 2015 & 40 & 2.4 & 15 & \\
March 3, 2015 & 38 & 1.6 & 30 & \\
\hline
\end{tabular}

deficiency. When our case is compared with the two patient cases that required vitamin $\mathrm{K}$ antagonist discontinuation, our patient had a more mild FVII deficiency and maintained higher FVIIc activity during anticoagulation therapy. For patients with lower FVIIc at baseline or who experience bleeding events or significant decreases in FVIIc with warfarin therapy, alternative therapies could be considered.

Our patient was initially treated with warfarin in 2011. At that time, evidence-based practice guidelines recommended long-term antithrombotic therapy with either warfarin or aspirin, since the patient had only one major risk factor-hypertension [21, 22]. In 2014, however, updated guidelines now favoured anticoagulation therapy for our patient, despite no change in her risk factors $[23,24]$. The American Heart Association/American College of Cardiology/Heart Rhythm Society (AHA/ACC/ HRS) 2014 guidelines recommend using the $\mathrm{CHA}_{2} \mathrm{DS}_{2^{-}}$ $\mathrm{VAS}_{\mathrm{c}}$ scoring system to assess stroke risk. The $\mathrm{CHA}_{2} \mathrm{DS}_{2^{-}}$ $\mathrm{VAS}_{\mathrm{c}}$ score for our patient was 3, due to hypertension, female sex, and age $\geq 65$ years. For scores $\geq 2$, anticoagulation therapy is recommended (warfarin: level of evidence-A; dabigatran, rivaroxaban, apixaban: level of evidence-B) [24]. These changes in treatment guidelines are significant for our patient, as aspirin is no longer considered an acceptable treatment option on the basis of her risk factors.

The new agents mentioned above represent a new group of anticoagulants known as direct oral anticoagulants (DOACs). This group currently includes direct thrombin (factor II [FII]) inhibitors (dabigatran) and direct FXa inhibitors (rivaroxaban, apixaban and edoxaban). The mechanisms by which both classes of agents act target a clotting factor only in the common pathway of the coagulation cascade, independent from FVII. Laboratory analysis has shown that these agents do not inhibit FVII $[25,26]$. In contrast, warfarin inhibits FVII in the extrinsic pathway. Clinical studies have shown dabigatran, rivaroxaban, apixaban and edoxaban to be comparably safe and effective in comparison with warfarin therapy for the treatment of atrial fibrillation, although patients with FVII deficiency and other RBDs were excluded from the trials [27-30]. However, because these agents provide therapeutic anticoagulation by targeting clotting factors below FVII in the clotting cascade (Fig. 1) and do not directly affect FVII activity, they may theoretically carry less bleeding risk than warfarin in patients with FVII deficiency.

This suggestion to choose an anticoagulant that does not affect FVII activity is supported by the case report [20] in 2009, which emphasized heparin use in patients with FVII deficiency. In that case, LMWH was safely tolerated throughout the duration of maintenance therapy. However, at that time, the DOACs were not approved or available for use. Interestingly, the DOACs have mechanisms of action similar to those of heparin, targeting FXa and/or FII. While heparin remains a viable option, it must be administered parenterally, which is a disadvantage in comparison with orally administered medications. One important difference between LMWH and the DOACs is the ability to monitor the anticoagulant effect beyond the FVIIc activity assay, as LMWH can be monitored using anti-Xa levels, which correlate with the actual bleeding risk. This ability (not requirement) to monitor the anticoagulant effect may serve as an advantage in this population at higher risk of bleeding, as it gives the clinician a method to objectively assess the degree of anticoagulation being provided. The DOACs rivaroxaban, apixaban and edoxaban are FXa inhibitors and, although anti-Xa level monitoring is not performed clinically, it would seem reasonable that it could be utilized 
with these agents as well [31]. Lastly, the initial lack of antidotes or reversal agents for the DOAC class was considered to be a disadvantage but, recently, highly specific reversal agents have been developed and studied with positive results in clinical trials [32, 33]. Before reversal agents were available, a subgroup analysis from the ROCKET-AF trial of 779 patients who experienced major bleeding revealed no differences in outcomes in patients treated with rivaroxaban versus warfarin, including death (20.4 vs $26.1 \%$; hazard ratio $0.69,95 \%$ confidence interval 0.46-1.04). There was also less use of prothrombin complex concentrate (PCC) and significantly less use of fresh frozen plasma (FFP) with rivaroxaban compared with warfarin [34]. This information supports the consideration of DOACs as a potential alternative to vitamin $\mathrm{K}$ antagonists, even in unique and high-risk patient situations.

\section{Conclusion}

Warfarin has been the treatment of choice for thromboembolic conditions for multiple decades, but the data are limited in patients with RBDs. We have described a successful re-challenge of warfarin therapy in a patient with FVII deficiency. However, compared with our patient case, patients with more severe FVII deficiency at baseline or those who experience lower levels of FVII activity during warfarin therapy may not observe the same positive response. The emergence of the DOAC group of anticoagulants has provided more options for clinicians when treating patients with RBDs in the outpatient setting. Based on their mechanisms of action in relation to the coagulation cascade, it would seem DOACs could potentially be safer alternatives to warfarin for patients with FVII deficiency by providing similarly therapeutic anticoagulation with less bleeding risk. Future studies would be helpful to evaluate the safety and effectiveness of DOAC therapy in patients with FVII deficiency. However, because of the rare nature of the disorder, it is not feasible to conduct prospective, randomized studies. Large clinical trials of anticoagulants typically exclude patients with FVII deficiency and other RBDs, although this would be an excellent way to obtain more data. It is imperative for clinicians to publish case reports and other retrospective data to guide future therapy in this population.

Acknowledgments Eric Paulus, Kathy Komperda, Gabriel Park and Julie Fusco declare they have no conflict(s) of interest. No financial support was received for the preparation of this manuscript.

\section{Compliance with Ethical Standards}

Written informed consent was obtained from the patient for publication of this case report and any accompanying images. A copy of the written consent may be requested for review from the corresponding author.

Funding The authors received no sources of funding that require acknowledgment.

Open Access This article is distributed under the terms of the Creative Commons Attribution-NonCommercial 4.0 International License (http://creativecommons.org/licenses/by-nc/4.0/), which permits any noncommercial use, distribution, and reproduction in any medium, provided you give appropriate credit to the original author(s) and the source, provide a link to the Creative Commons license, and indicate if changes were made.

\section{References}

1. Konkle B. Bleeding and thrombosis. In: Longo DL, Kasper DL, Jameson JL, Fauci AS, Hauser SL, Loscalzo J, editors. Harrison's principles of internal medicine. 18th ed. New York: McGrawHill; 2012. p. 457-64.

2. Lawson LH, Kalafatis M, Stram S, et al. A model for the tissue factor pathway to thrombin. J Biol Chem. 1994;269:23357-66.

3. Broze GJ Jr. Tissue factor pathway inhibitor and the revised hypothesis of blood coagulation. Annu Rev Med. 1995;46:103-12.

4. Bolton-Maggs PH. The rare inherited coagulation disorders. Pediatr Blood Cancer. 2013;60:S37-40.

5. Ruiz-Saez A. Occurrence of thrombosis in rare bleeding disorders. Semin Thromb Hemost. 2013;39:684-92.

6. World Federation of Hemophilia. Report on the annual global survey. Montreal: World Federation of Hemophilia; 2010. p. $1-45$.

7. Lapecorella M, Marian G. Factor VII deficiency: defining the clinical picture and optimizing therapeutic options. Haemophilia. 2008; $14: 1170-5$

8. Fair DS. Quantitation of factor VII in the plasma of normal and warfarin-treated individuals by radioimmunoassay. Blood. 1983;62:784-91.

9. Morrissey JH, Macik BG, Neuenschwander PF, Comp PC. Quantitation of activated factor VII levels in plasma using a tissue factor mutant selectively deficient in promoting factor VII activation. Blood. 1993;81:734-44.

10. Goudemand J, Caron C, Dreyfus M, Sie P. Standardization of factor VII/activated factor VII measurement in plasma of patients treated with recombinant factor activated VII. Blood Coagul Fibrinolysis. 2003;14:505-11.

11. Peyvandi F, Bolton-Maggs PHB, Batorova A, et al. Rare bleeding disorders. Haemophilia. 2012;18:148-53.

12. Mariani G, Herrmann FH, Dolce A, et al. Clinical phenotypes and factor VII genotype in congenital factor VII deficiency. Thromb Haemost. 2005;93:481-7.

13. Benlakhal F, Mura T, Schved JF, et al. A retrospective analysis of 157 surgical procedures performed without replacement therapy in 83 unrelated factor VII-deficient patients. J Thromb Haemost. 2011;9:1149-56

14. Marty S, Barro C, Chatelain B, et al. The paradoxical association between inherited factor VII deficiency and venous thrombosis. Haemophilia. 2008;14:564-70.

15. Paul B, Oxeley A, Brigham K, Cox T, Hamilton PJ. Factor II, VII, IX, and X concentrations in patients receiving long term warfarin. J Clin Pathol. 1987;40:94-8.

16. Baruch L. Laboratory monitoring of anticoagulant medications: focus on novel oral anticoagulants. Postgrad Med. $2013 \cdot 125 \cdot 135-45$ 
17. Christensen TD, Jensen C, Larsen TB, Christiansen K, Sorensen B. Thrombin generation and coagulation factor activities: evaluation and comparison with the international normalized ratio. Blood Coagul Fibrinolysis. 2009;20:358-65.

18. Davidson SJ, Turner N, Tillyer L. Anticoagulation of a patient with hypertrophic cardiomyopathy and factor VII deficiency. Blood Coagul Fibrinolysis. 2010;21:707-8.

19. Baltodano LE, Mungall DR, Watson DD. Management of a patient with atrial fibrillation and factor VII deficiency. Ann Pharmacother. 1998;32:1251-2.

20. Arellano-Rodrigo E, Gironella M, Nicolau I, et al. Clinical management of thrombosis in inherited factor VII deficiency: a description of two cases. Thromb Haemost. 2009;101:402-4.

21. Singer DE, Albers GW, Dalen JE, et al. Antithrombotic therapy in atrial fibrillation: American College of Chest Physicians evidence-based clinical practice guidelines (8th edition). Chest. 2008; 133:546S-92S.

22. Fuster V, Rydén LE, Cannom DS, et al. ACC/AHA/ESC 2006 guidelines for the management of patients with atrial fibrillation-executive summary: a report of the American College of Cardiology/American Heart Association Task Force on Practice Guidelines and the European Society of Cardiology Committee for Practice Guidelines. Circulation. 2006;114:700-52.

23. You JJ, Singer DE, Howard PA, et al. Antithrombotic therapy for atrial fibrillation: antithrombotic therapy and prevention of thrombosis, 9th ed: American College of Chest Physicians evidence-based clinical practice guidelines. Chest. 2012;141:e531S$75 \mathrm{~S}$.

24. January CT, Wann LS, Alpert JS, et al. 2014 AHA/ACC/HRS guideline for the management of patients with atrial fibrillation: executive summary. Circulation. 2014;64:2246-80.
25. Perzborn E, Strassburger J, Wilmen A, et al. In vitro and in vivo studies of the novel antithrombotic agent BAY 59-7939 - an oral, direct factor Xa inhibitor. J Thromb Haemost. 2005;3:514-21.

26. Pinto DJ, Orwat MJ, Koch S, et al. Discovery of 1-(4-methoxyphenyl)-7-oxo-6-(4-(2-oxopiperidin-1-yl)phenyl)-4,5,6,7-tetrahydo-1H-pyrazolo[3,4-c]pyridine-3-carboxamide (apixaban, BMS-562247), a highly potent, selective, efficacious, and orally bioavailable inhibitor of blood coagulation factor Xa. J Med Chem. 2007;50:5339-56.

27. Connolly SJ, Ezekowitz MD, Yusuf S, et al. Dabigatran versus warfarin in patients with atrial fibrillation. $\mathrm{N}$ Engl $\mathrm{J}$ Med. 2009;361:1139-51.

28. Patel MR, Mahaffey KW, Garg J, et al. Rivaroxaban versus warfarin in nonvalvular atrial fibrillation. $\mathrm{N}$ Engl $\mathrm{J}$ Med. 2011;365:883-91.

29. Granger CB, Alexander JH, McMurray JJV, et al. Apixaban versus warfarin in patients with atrial fibrillation. $\mathrm{N}$ Engl $\mathrm{J}$ Med. 2011;365:981-92.

30. Giugliano RP, Ruff CT, Braunwald E, et al. Edoxaban versus warfarin in patients with atrial fibrillation. $\mathrm{N}$ Engl J Med. 2013;369:2093-104.

31. Samama MM, Guinet C. Laboratory assessment of new anticoagulants. Clin Chem Lab Med. 2011;49:761-72.

32. Pollack CV, Reilly PA, Eikelboom J, et al. Idarucizumab for dabigatran reversal. N Engl J Med. 2015;373:511-20.

33. Siegal DM, Curnutte JT, Connolly SJ, et al. Andexanet alfa for the reversal of factor xa inhibitor activity. $N$ Engl $J$ Med. 2015;373:2413-24.

34. Piccini JP, Garg J, Patel MR, et al. Management of major bleeding events in patients treated with rivaroxaban vs warfarin: results from the ROCKET-AF trial. Eur Heart J. 2014;35:1873-80. 